\title{
EVALUATION OF THE JOINT BASED ON DIFFERENT SURFACE CONDITIONS FOR ALUMINUM-POLYAMIDE LASER WELDING
}

Paper \#

\author{
Mahdi Amne Elahi ${ }^{1}$, Marcus Koch ${ }^{2}$, Peter Plapper ${ }^{1}$ \\ ${ }^{1}$ University of Luxembourg, 6, rue Coudenhove-Kalergi, L-1359, Luxembourg \\ ${ }^{2}$ INM - Leibniz Institute for New Materials Campus D2 2, 66123 Saarbrücken, Germany
}

\begin{abstract}
Laser welding is one of the most promising joining techniques to realize hybrid joints between metals and polymers in order to achieve weight reduction and functionalization of the parts. The surface treatment of the metal has a decisive effect on the joint quality and thus on the mechanical properties. In the present study, different mechanical and laser-based surface treatments have been investigated to develop diverse surface conditions on aluminum. Abrasive blasting and laser ablation were used to increase the surface roughness, while abrasive polishing and laser polishing were applied to minimize the surface roughness. In contrast to abrasive surface treatments, laser-based ones were implemented to create artificial oxide layers on the aluminum surface. The surface structures of pretreated samples have been studied with scanning electron microscopy and roughness test. The laser welding of pretreated aluminum with polyamide was achieved with the heat conduction joining technique. To enlarge the welding area and control the heat input, spatial and temporal modulations of the laser beam were implemented. Finally, a single lap tensile-shear test, microscopic analysis of fractured surfaces, and welding cross-sections were employed to evaluate the joints. Results show that the presence of an artificial aluminum oxide layer and low roughness are essential to achieve a superior joint between aluminum and polyamide (improvement of approximately $58 \%$ in the shear load of the joint compared to as-received welded samples). The cross-section of the superior joint which is the laserpolished aluminum welded to polyamide is studied with transmission electron microscopy.
\end{abstract}

Keywords: Laser-assisted aluminum-polyamide welding, surface treatment, abrasive blasting, laser polishing, laser ablation.

\section{Introduction}

There are several joining techniques to realize the hybrid metal/polymer assemblies with the aim of weight reduction. Among them, laser welding has got enormous attention thanks to its unique advantages like exceptional control over heat input and automated capabilities $\left[{ }^{1},{ }^{2}\right]$. Katayama and his team introduced the laser-assisted metals to polymers welding [ $\left.{ }^{3}\right]$. Currently, based on the transparency of the polymeric partner to the wavelength of the laser beam, there are two main techniques for laser welding of metals to polymers. If the polymer has high transparency, the direct laser joining technique can be implemented. Otherwise, the conduction joining technique is more promising to achieve the joint $\left[{ }^{3},{ }^{4}\right]$. Several publications reported on the effect of surface treatments to improve the joining quality between metals and polymers in laser welding $\left[{ }^{5},{ }^{6},\right]$. However, due to the lack of comprehensive understanding of the bonding and failure mechanisms and thus unreliability of the joints, the laser metals to polymers welding is not well industrialized.

For aluminum and polyamide laser-welded specimens, without applying the pretreatment specifically on the aluminum surface, the mechanical properties of the joints are relatively low and the failure always happens in a mixed adhesive/cohesive mode near the interface of metal/polymer. Also, welding with excessive energy input results in the degradation of materials specifically polyamide at the weld zone which is detrimental to the joint quality and the mechanical properties $\left.{ }^{8}\right]$. Promoting the physicochemical bonding at the interface of metal/polymer can improve the bonding quality and long-term durability of the joints $\left[{ }^{9}\right]$. Therefore, it is essential to select an appropriate pretreatment for the aluminum surface to ensure the superior mechanical properties of the joint.

For this study, it is tried to implement different surface treatments on the aluminum surface and weld them to polyamide with similar welding parameters. As the welding parameters (e.g. heat input and clamping pressure, materials properties) are similar for all samples, increasing the surface roughness will decrease the contact area between the weldments and thus will increase the thermal contact resistance $\left[{ }^{10}\right]$. Investigation on the mechanical properties of the joints 
followed by the mechanism of failure shows the effectiveness of mechanical interlocking or physicochemical bonding for the present material combination.

\section{Experimental Procedure}

For the experiments, $30 \times 60 \times 0.5 \mathrm{~mm}$ sheets of aluminum 1050-H24 (will be addressed as $\mathrm{Al}$ ) and $25 \times 75 \times 4 \mathrm{~mm}$ polyamide 6.6 (will be addressed as PA) were used. The PA samples were conditioned based on the ISO-1110 standard to control the humidity content. All samples were wiped with ethanol directly before processing.

The laser ablation processes were done with a pulsed wave TruMark 6130 laser machine with a wavelength of $1064 \mathrm{~nm}$, beam spot size of $28 \mu \mathrm{m}$, and beam quality of $\mathrm{M}^{2}<1.2$. The laser polishing process and laser welding were implemented with a continuous wave fiber laser (TruFiber 400) with a wavelength of $1070 \mathrm{~nm}$ and equipped with Scanlab HS20 2D f- $\Theta$ scanner head to achieve a beam quality of $\mathrm{M}^{2}=1.03$ and the spot size of $30 \mu \mathrm{m}$. The laser ablation process was implemented to achieve two levels of surface roughness (medium and high Ra). For the laser polishing under Argon (Ar) (purity of 99.995\%), the process was implemented in an enclosed chamber covering the sample and the laser scanner. The Ar gas was flowed into the chamber with constant pressure for a few minutes before and during the process. The parameters of the laser polishing and the laser ablation processes are covered in table 1.

Table 1. The parameters of the laser ablation and the laser polishing processes.

\begin{tabular}{|c|c|c|c|c|c|c|}
\hline & 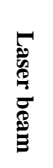 & 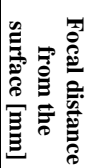 & 쥴 & 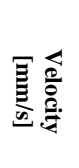 & 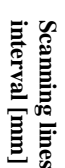 \\
\hline \multirow{2}{*}{$\begin{array}{c}\begin{array}{c}\text { Laser } \\
\text { ablation }\end{array} \\
\end{array}$} & Medium Ra & $\mathrm{PW}$ & 0 & 120 & 1000 & 0.05 \\
\hline & high Ra & PW & 0 & 60 & 145 & 0.07 \\
\hline \multicolumn{2}{|c|}{ Laser polishing } & $\mathrm{CW}$ & +3 & & 2000 & 0.05 \\
\hline
\end{tabular}

Like the laser ablation process, abrasive blasting was implemented to achieve two Ra levels; silicon carbide and polyamide powder were used as blasting media to provide high and medium Ra respectively. As will be seen in the results and discussions section, for each level of $\mathrm{Ra}$ (medium and high), the roughness values are comparable for both processes. The abrasive polishing process was applied by polishing the as-received $\mathrm{Al}$ surface with diamond suspensions. It is worth mentioning that after abrasive blasting and abrasive polishing processes, the samples were cleaned with an ultrasonic ethanol bath for 10 minutes to avoid undesirable contaminations.

The laser welding process was achieved by applying the laser beam to the $\mathrm{Al}$ surface in an overlapping geometry with PA below (conduction joining process). Clamping pressure is provided with four toggle clamps and it is fixed for all samples. Figure 1 shows the spatial and temporal modulations of the laser beam, which are considered to control the heat flow from Al to PA effectively. The modulated power of $260 \mathrm{~W}$ was selected based on pre-tests to avoid the thermal pyrolysis of PA $\left[^{8,11}\right]$.

To evaluate the mechanical properties of the joints, the welded specimens were tested in a single lap tensileshear configuration with a constant speed of $2.5 \mathrm{~mm} / \mathrm{s}$. Figure 2 depicts the schematics of the test. The supports were used to limit the bending of the PA samples during the test. The reported values are representative of at least five measurements.

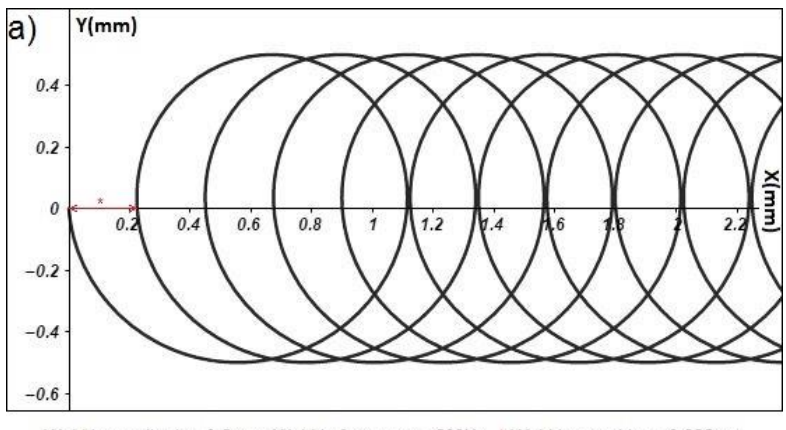

Wobble amplitude $=0.5 \mathrm{~mm}$, Wobble frequency $=500 \mathrm{~Hz},{ }^{*}$ Wobble repetition $=0.225 \mathrm{~mm}$

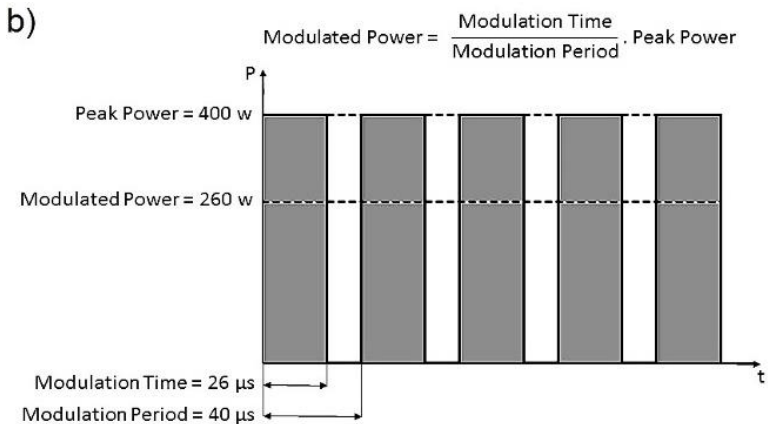

Figure 1. a) Spatial modulation and b) temporal modulation of the laser beam "Reproduced from Amne Elahi, M., Koch, M., Plapper, P., Laser polishing of Aluminum and Polyamide for dissimilar laser welded assemblies, Lasers in Manufacturing Conference (LiM) 2019."

To study the samples' surface structure and chemistry for both the pretreatment processes and the fracture surfaces, a Scanning Electron Microscope (FEI ESEM Quanta 400 FEG) equipped with an Energy-Dispersive X-ray Spectroscopy (EDS) (EDAX Genesis V6.04) was used. The results consist of Secondary Electrons (SE) 
and Backscattered Electrons (BSE) images in addition to the local chemical analysis done EDS. The surface roughness was also studied by roughness measurement based on ISO 4288-1996 standard. The reported values are representative of at least five measurements consist of $\mathrm{Ra}$ (arithmetical mean roughness value), $\mathrm{Rz}$ (mean roughness depth), and RSm (mean peak width).

Finally, for the laser-polished Al samples welded to PA, a cross-sectional analysis of the bonding area was made by Focused Ion Beam (FIB) preparation and Transmission Electron Microscopy (TEM) imaging. For this purpose, an $\mathrm{Al}$ fractured surface was used meaning that the PA was released from the Al substrate, and a thin layer of PA on top of the laser-polished $\mathrm{Al}$ was chosen for TEM lamella preparation by FIB FEI Versa 3D. Firstly, a thin platinum (Pt) layer was deposited on top of the PA using the electron beam followed by a Pt deposition by the Gallium (Ga) ion beam. Then a crosssection was made using the $\mathrm{Ga}$ ion beam at $30 \mathrm{kV}$ accelerating voltage. The TEM lamella was prepared by transferring the lamella to a copper grid. Lastly, the TEM lamella was thinned carefully by cleaning the specimen using $\mathrm{Ga}$ ions at $5 \mathrm{kV}$ accelerating voltage. Subsequently, TEM bright field imaging at $200 \mathrm{kV}$ accelerating voltage was performed using a JEOL JEM$2100 \mathrm{LaB}_{6}$ transmission electron microscope with an inbuilt Gatan Orius SC1000 CCD camera.

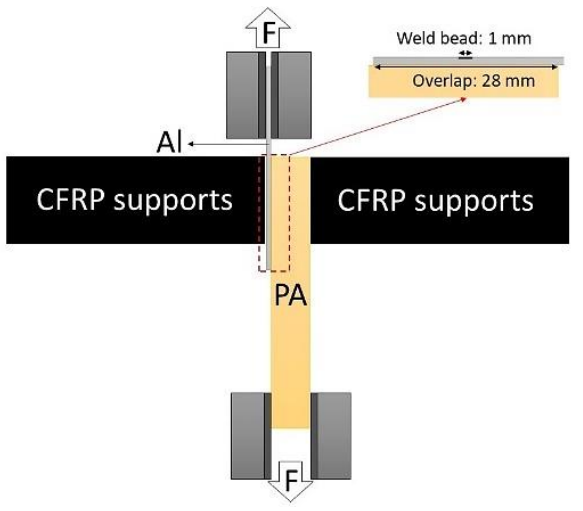

Figure 2. Schematics of the tensile-shear test.

\section{Results and Discussions}

Figures 3 and 4 depict Ra, Rz, and RSm measurements for different Al surface treatments. As can be observed, for $\mathrm{Ra}$ the values can be categorized into four main groups; as-received condition with anisotropic surface roughness values, medium and high Ra for laser-ablated and abrasive-blasted samples, and low $\mathrm{Ra}$ values for laser and abrasive-polished samples. Applying the surface treatment processes on as-receive $\mathrm{Al}$ results in more isotropic roughness except for RSm values for laser-polished and laser-ablated medium Ra samples.
The anisotropy of RSm values due to the scanning lines for the mentioned samples can be observed in the corresponding microscopic images.

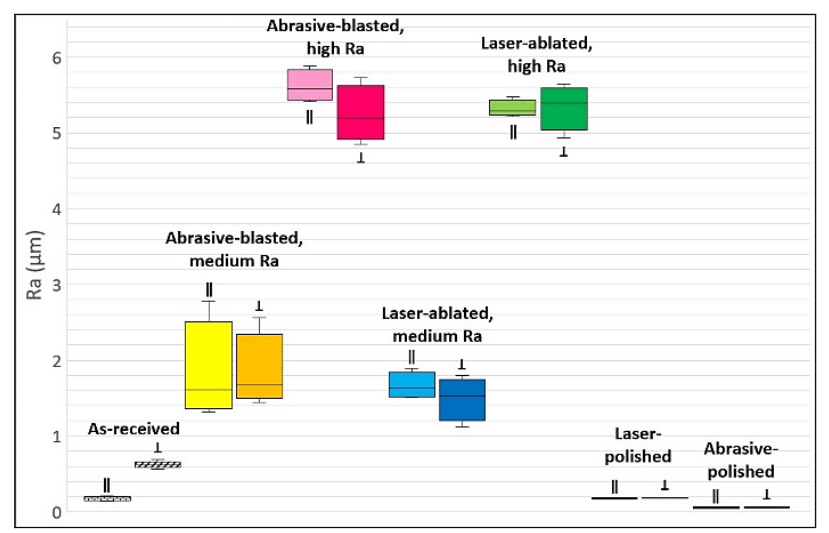

Figure 3. Ra measurement for different surface treatments (II: rolling direction, $\mathbf{\perp}$ : orthogonal to the rolling direction).

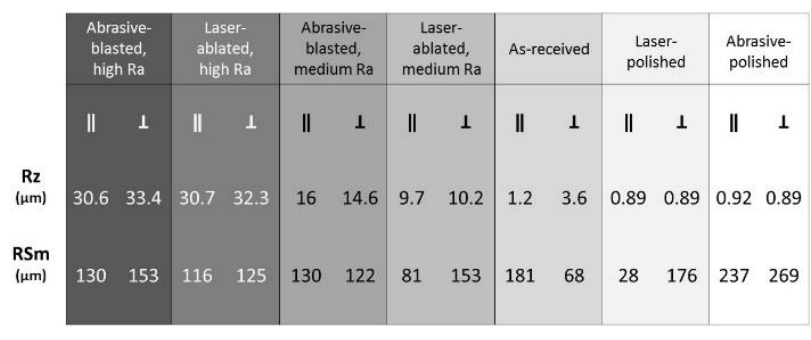

Figure 4. Rz and RSm measurements for different surface treatments (II: rolling direction, $\mathbf{\perp}$ : orthogonal to the rolling direction).

Figure 5 shows the microscopic image and the corresponding EDS analysis of the as-received surface. Based on a previous study, the as-received Al surface is covered by a natural $\mathrm{Al}$ oxide with a thickness of approximately $25 \mathrm{~nm}$. The natural $\mathrm{Al}$ oxide is quite dense $\left[{ }^{8}\right]$.

Figure 6 shows the SEM images of the laser-polished sample under the atmospheric environment with the EDS analysis. The scanning lines of the polishing process are visible in figure 6-a which results in anisotropic $\mathrm{RSm}$ in contrast to $\mathrm{Ra}$ and $\mathrm{Rz}$ values (figures 3 and 4). Higher magnification of the surface shows that a nanostructure oxide is created on the surface by the laser polishing process. The artificial oxide layer has approximately $1.5 \mu \mathrm{m}$ thickness and it is considerably porous compare to the natural $\mathrm{Al}$ oxide $\left.{ }^{8}\right]$. The higher oxygen content of laser-polished $\mathrm{Al}$ compared to asreceived one is explained by the significant difference in artificial and natural $\mathrm{Al}$ oxide thicknesses. It is worth mentioning that the laser-polishing process has a surface 
cleaning effect, which is observed by the lower carbon content of the laser-polished sample.

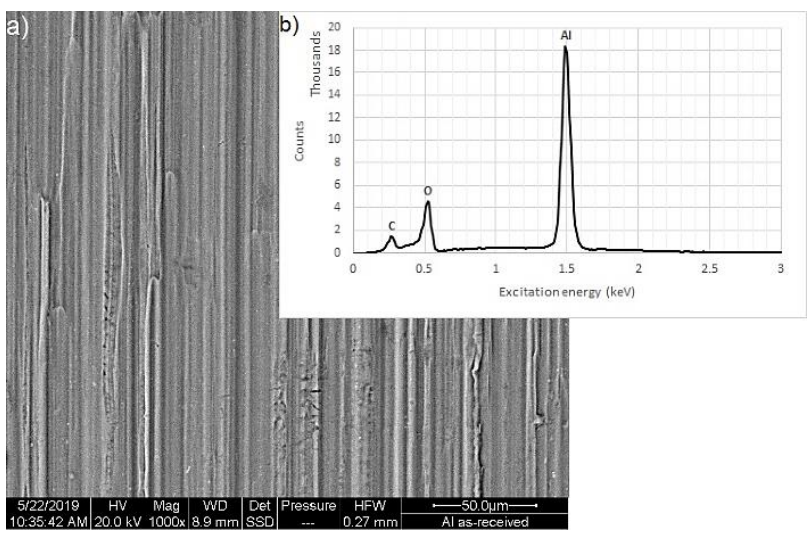

Figure 5. a) SEM image of the as-received surface and b) the corresponding EDS analysis.
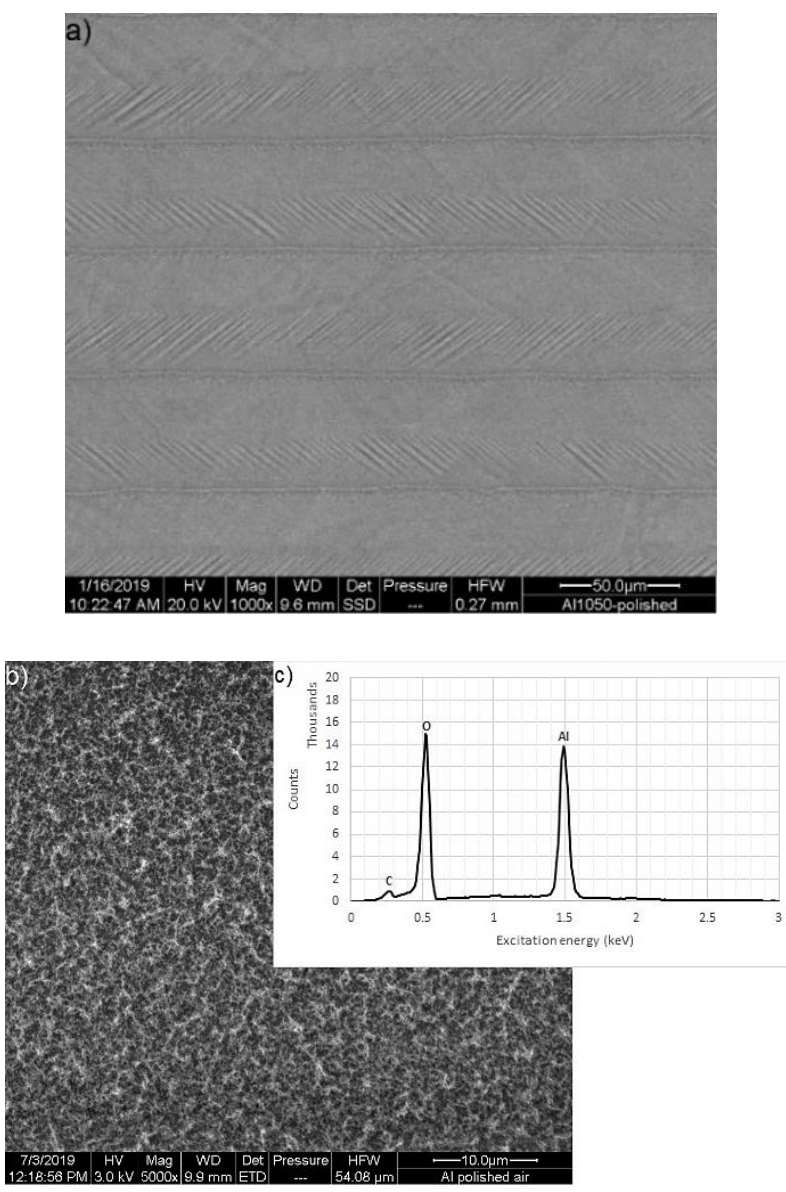

Figure 6. a) SEM image of the laser-polished surface under atmospheric environment, b) higher magnification of the same sample, and c) the corresponding EDS analysis.
Figure 7 depicts the SEM images of the laser polished samples under the Ar atmosphere. In contrast to the samples laser-polished under the atmospheric environment, the development of artificial oxide is considerably suppressed under the Ar environment. The EDS analysis of the artificial oxide particles is comparable to that shown in figure 6-b, while the EDS analysis of the Al matrix is similar figure 5-b with less carbon content because of the cleaning effect of the laser polishing process (data not shown).

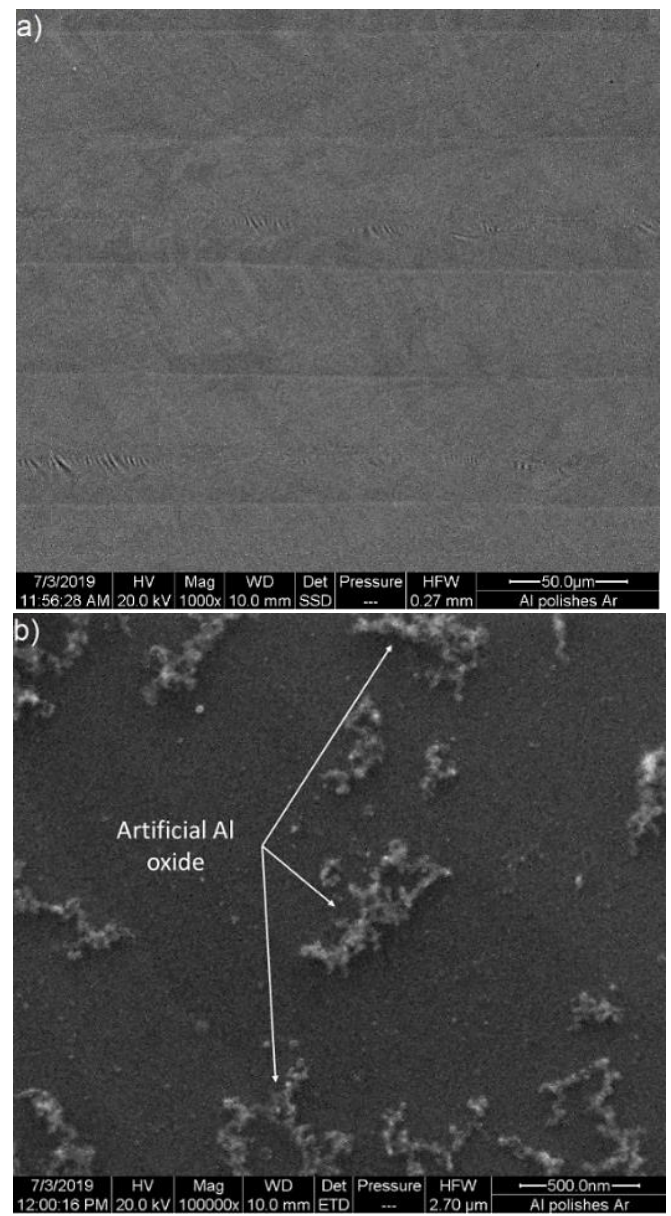

Figure 7. a) SEM image of the laser-polished surface under Ar atmosphere, b) higher magnification of the same sample.

Figure 8 depicts the SEM images of the laser-ablated samples to achieve medium and high levels of roughness. The EDS analysis of the laser-ablated samples (data not shown) shows a higher level of oxygen contents compared to the as-received condition, which is an indication of the presence of an artificial oxide layer. The surface chemistry of the laser-ablated high $\mathrm{Ra}$ samples is less uniform compared to the corresponding medium Ra samples. It is due to different process parameters especially lower pulse frequency. 
The scanning lines and therefore anisotropy of RSm value are visible for laser-ablated medium Ra samples.

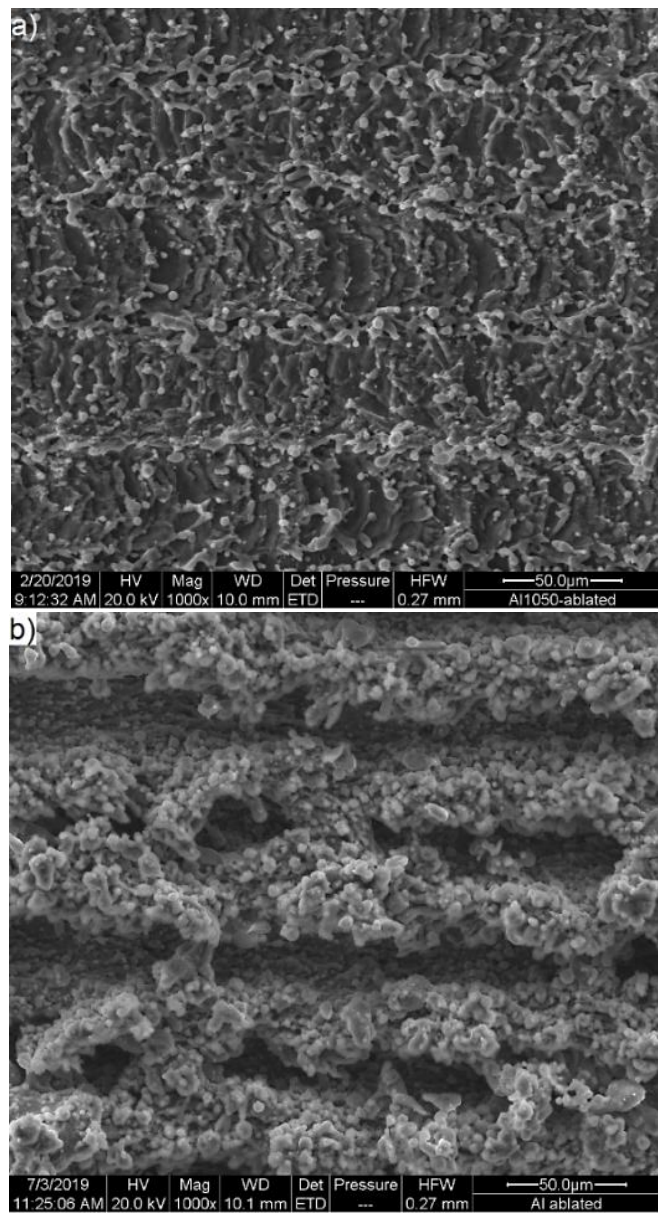

Figure 8. SEM images of laser-ablated surfaces, a) medium roughness, and $b$ ) high roughness.

Figure 9 shows the SEM images of abrasive-blasted samples with two levels of surface roughness. Each category is comparable to a corresponding laser-ablated one regarding surface roughness values, however; the EDS analysis (data not shown) is comparable to the asreceived condition. The natural $\mathrm{Al}$ oxide is present on abrasive-blasted surfaces with higher surface roughness compared to the as-received condition.

Figure 10 shows shear load values for different pretreated $\mathrm{Al}$ samples welded to PA. As the welding parameters are similar for the samples, it is tried to link the shear loads of the joint to the surface condition of $\mathrm{Al}$ samples. The as-received samples with the mean shear load of approximately $1018 \mathrm{~N}$ are the reference. In this case, the welding path is parallel to the rolling direction of $\mathrm{Al}$ samples. By changing the $\mathrm{Al}$ samples orientation, (the welding path is perpendicular to the rolling direction) the shear loads of the joints are comparable to the previous setup (approximately $1014 \mathrm{~N}$ on average).
It is the first indication that the mechanical interlocking is not the dominant bonding mechanism between $\mathrm{Al}$ and PA in the laser-assisted welding process.
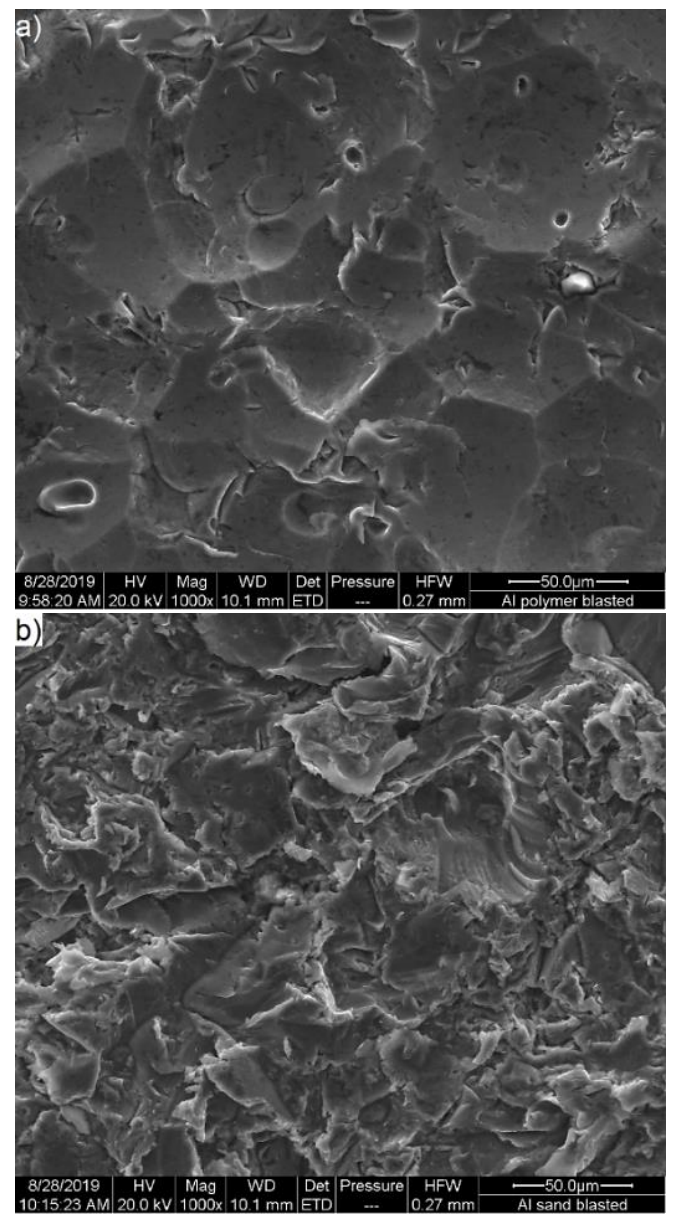

Figure 9. SEM images of abrasive-blasted surfaces, a) medium roughness, and b) high roughness.

By making the $\mathrm{Al}$ surface rough with the presence of the natural oxide layer, a huge drop in the shear loads of the joint can be observed. In the extreme case, which is abrasive-blasted high Ra, there is practically no durable joint. Welding these samples even with higher modulated power (up to $300 \mathrm{~W}$ ) does not improve the shear load of the joints.

For laser-ablated samples, the shear loads were improved compared to the corresponding blasted category. Therefore, to increase the shear load of the joints, the development of an artificial oxide layer is significantly more effective rather than an increase of surface roughness. In fact, for the high Ra values of both categories, the shear load is lower than that of medium $\mathrm{Ra}$ due to higher thermal contact resistance. Higher Ra values bring higher thermal contact resistance; therefore, heat flow from $\mathrm{Al}$ to PA will be less uniform to create a reliable joint. However, considering laser- 
ablated medium $\mathrm{Ra}$ samples, there is a significant improvement in shear load compared to the as-received condition.

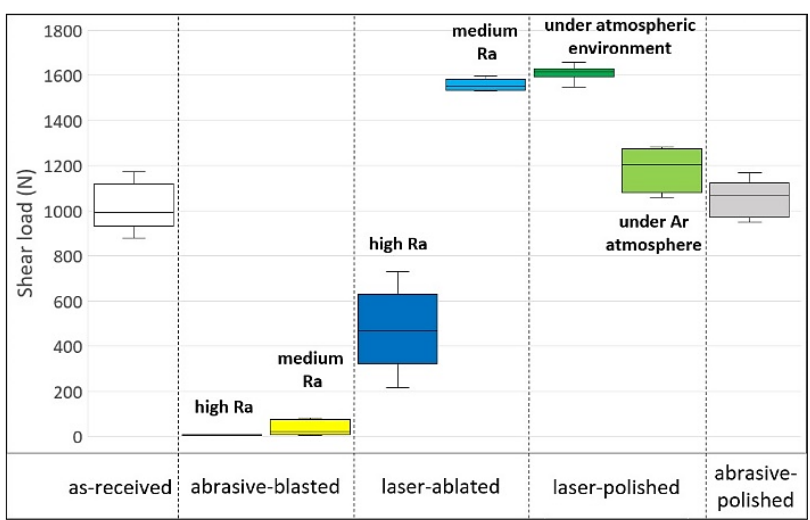

Figure 10. Shear load of the samples with different pretreatments.

By making a comparison between the shear loads of the samples with low $\mathrm{Ra}$ values (laser-polished and abrasive-polished), the importance of the artificial oxide layer will be better clarified. As presented in figures 6 and 7, the structure of the artificial oxide layer is under the control of the laser-polishing environment. With the significantly limited nano-structured oxide layer due to the $\mathrm{Ar}$ environment, the improvement of the shear load is low and more interestingly, the shear load of abrasivepolished samples is comparable to that of the asreceived sample. It indicates that the slight reduction of surface roughness (consequently lower thermal contact resistance) with the presence of natural $\mathrm{Al}$ oxide is not effective to increase the shear loads of the joints. The natural $\mathrm{Al}$ oxide is dense and does not provide infiltration sites for molten PA. However, considering the sample laser-polished under the atmosphere environment, the highest shear load is achieved thanks to the presence of a relatively thick artificial $\mathrm{Al}$ oxide layer, which can provide infiltration sited for the molten PA $\left[^{8}\right]$.

The slight difference of the mechanical properties and failure mechanisms between laser-polished under atmospheric environment and laser-ablated medium Ra samples has been studied separately and will be published accordingly. The failure of laser-ablated samples is due to the entrapped air bubbles in PA near the interface of $\mathrm{Al} / \mathrm{PA}$, while the failure of the laserpolished samples happens in the heat-affected zone (HAZ) of PA. It is located a few micrometers outside of the PA layer which is melted during the laser welding process $\left[{ }^{8}\right]$. To sum up, by applying the laser polishing process under the atmospheric environment and generation of a porous artificial oxide layer, the tensileshear load of the joint will be improved by approximately $58 \%$ compared to the as-received condition welded with similar parameters.

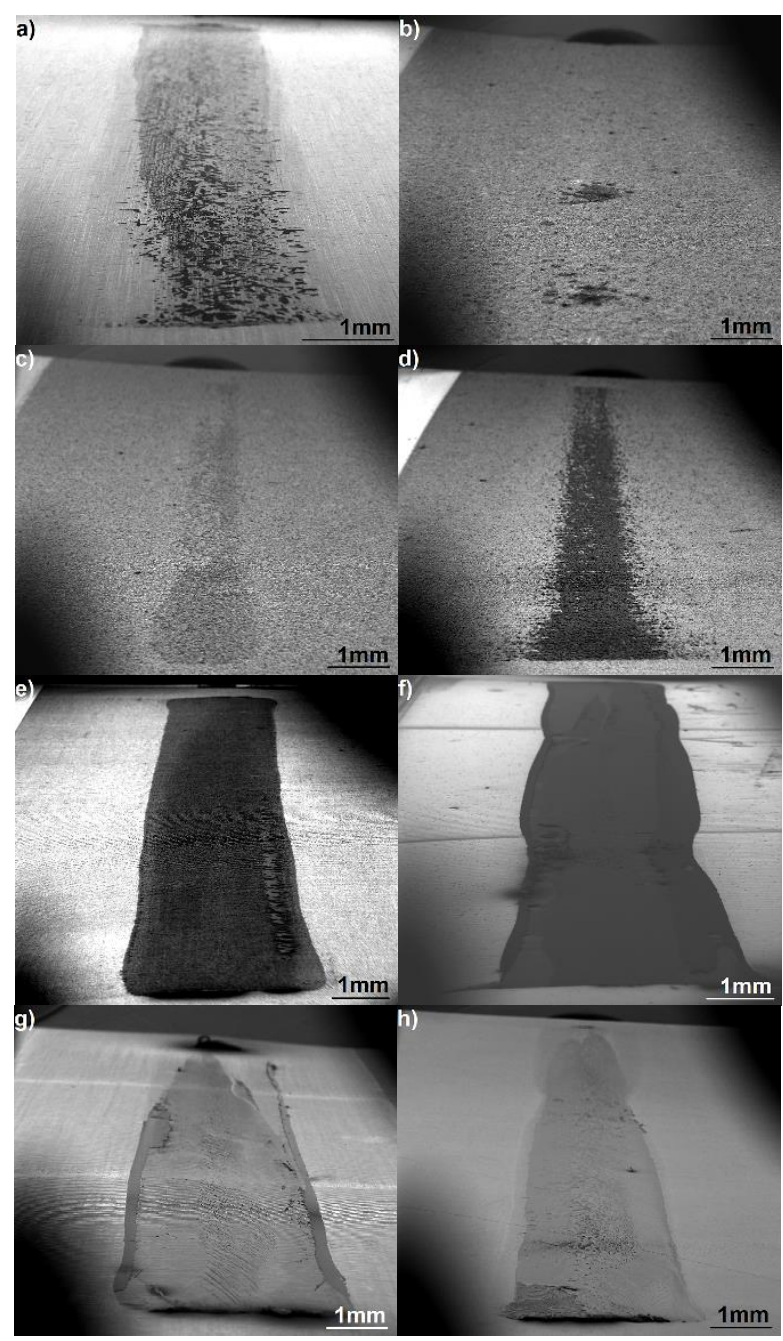

Figure 11. Al fracture surfaces in a tilted view, a) asreceived, b) abrasive-blasted high $\mathrm{Ra}, \mathrm{c}$ ) abrasiveblasted medium $\mathrm{Ra}$, d) laser-ablated high $\mathrm{Ra}$, e) laserablated medium Ra, f) laser-polished under atmospheric environment, g) laser-polished under $\mathrm{Ar}$ atmosphere, and h) abrasive-polished.

Figure 11 shows the $\mathrm{Al}$ fracture surfaces for different pretreatment processes. The PA residue on the $\mathrm{Al}$ surface represents the connection area between the materials. By making a comparison between figures 10 and 11, the morphology of PA residue on the Al surface after the mechanical failure can be explained by the corresponding mechanical properties. The PA residue on the as-received Al surface (figure 11-a) is not a uniform layer as the natural $\mathrm{Al}$ oxide cannot prove good wettability and thus adhesion for molten PA. Therefore, a mixed adhesive/cohesive failure is observed for asreceived samples. Increasing the surface roughness with 
the presence of natural $\mathrm{Al}$ oxide makes the PA residue significantly narrower due to higher thermal contact resistance. By creating an artificial $\mathrm{Al}$ oxide layer, the PA residue is significantly wider and thicker even though the thermal contact resistance is supposed to be similar to the corresponding abrasive-blasted samples. It is an indication that the artificial $\mathrm{Al}$ oxide layer can promote adhesion with PA.

In the case of the low roughness of laser-polished under $\mathrm{Ar}$ and abrasive polished samples, low contact resistance provides a better heat flow from $\mathrm{Al}$ to PA, therefore, results in wider PA residue. However, the PA residue has a relatively low thickness. Therefore, the anchoring effect is weak and as seen before, the shear load is not significantly improved compared to the asreceived sample as the formation of nano-structure $\mathrm{Al}$ oxide is significantly suppressed. The relationship between the thickness of the artificial Al oxide layer and the mechanical properties of the joint will be studied separately.

Considering the laser-polished under atmospheric environment sample, it represents a wide and relatively thick PA residue on the $\mathrm{Al}$ surface thanks to the artificial Al oxide layer. Therefore, in contrast to the samples with natural $\mathrm{Al}$ oxide, the high anchoring effect can be observed which results in the superior shear load between the samples. The bonding mechanism and identification of bonding type at the interface of metal/polymer will be studied later.

The bonding between laser-polished $\mathrm{Al}$ and PA after welding was investigated by TEM after FIB preparation of a thin TEM lamella, as shown in figure 12. An area where a thin layer of PA on top of the laser polished $\mathrm{Al}$ was chosen for FIB preparation depicted in figure 12-a. A cross-section was prepared using the $\mathrm{Ga}$ ion beam after Pt deposition (figure 12-b). Secondary electron imaging using the FIB shows a dense bonding between the laser-polished Al substrate and the PA (figure 12c,d). Bright-field TEM imaging can visualize this bonding in higher resolution (figure 12-e,h). Individual $\mathrm{Al}_{\mathrm{x}} \mathrm{O}_{\mathrm{y}}$ nanostructures still bound on the $\mathrm{Al}$ substrate can be found in the PA matrix (figure 12-f,h). Highresolution TEM bright field imaging cannot show any lattice fringes indicating that the PA matrix is amorphous (figure 12-h).

Therefore, the dense bonding of the PA matrix to the $\mathrm{Al}_{\mathrm{x}} \mathrm{O}_{\mathrm{y}}$ nanostructures on top of the laser-polished $\mathrm{Al}$ substrate can be observed which is enabled by a perfect wetting of PA during the welding process. No bubbles or voids could be found at the cross-section both by secondary electron and by bright-field TEM imaging. This implies that for these samples, the physicochemical bonding is promoted rather than the mechanical interlocking. Due to the high surface area of the $\mathrm{Al}_{\mathrm{x}} \mathrm{O}_{\mathrm{y}}$ nanostructures and the perfect wetting, the anchoring effect is promoted, and an extremely strong bond is formed, which results in a superior shear load during mechanical testing.
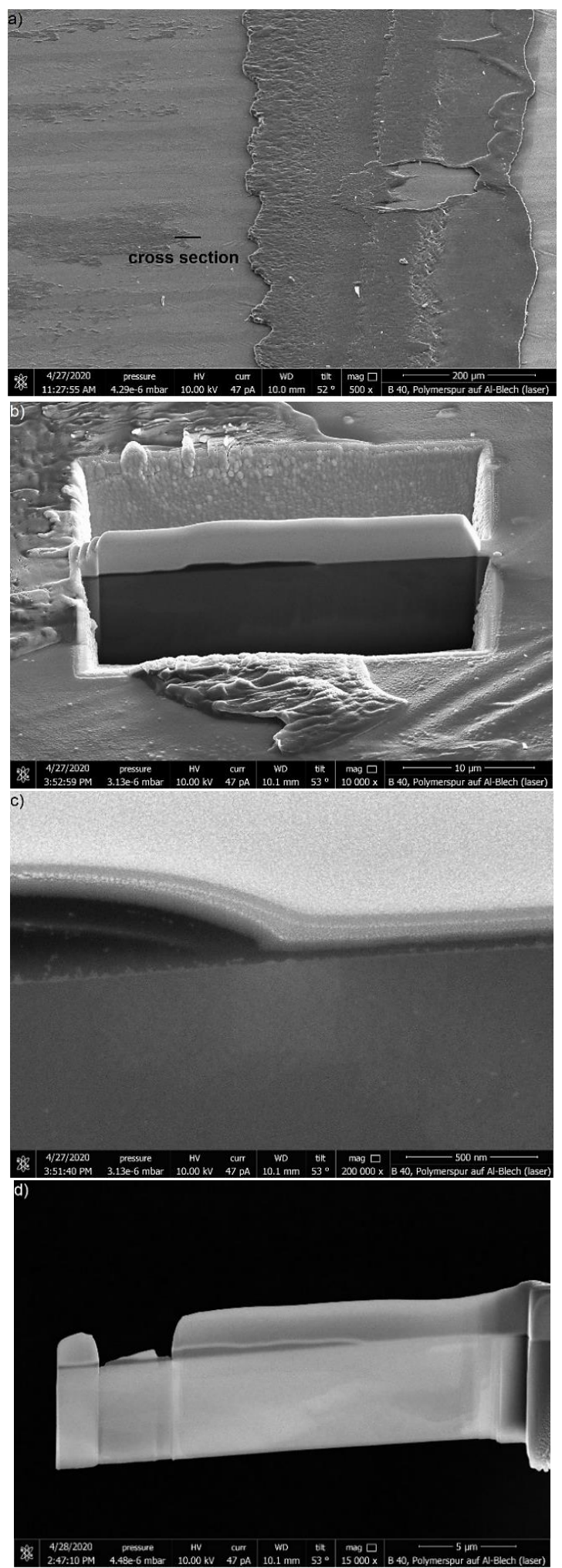


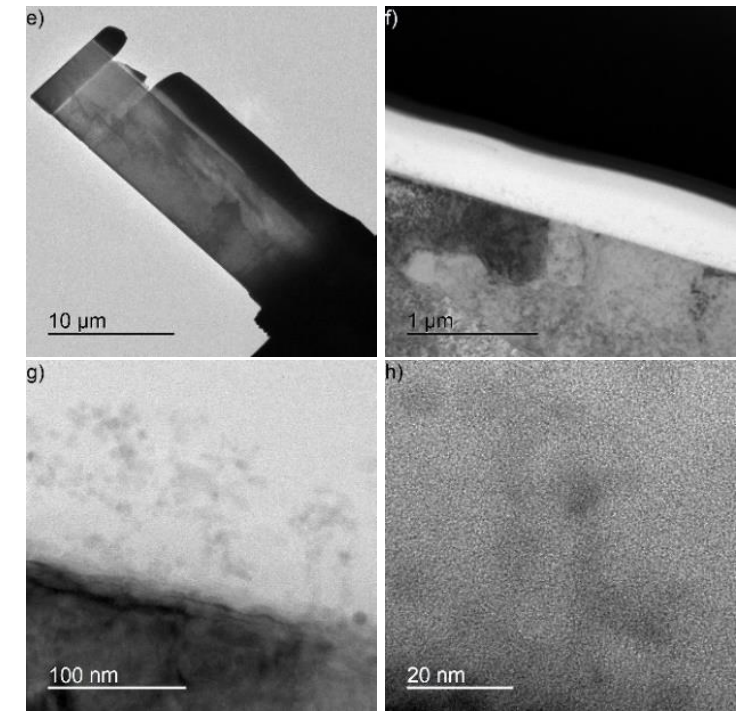

Figure 12. Al fracture surface a) place where the crosssection is made, $b$ ) cross-section after Pt deposition and $\mathrm{Ga}$ ion beam milling, c) secondary electron image of cross-section, d) secondary electron image of TEM lamella after transfer to the copper grid, e) bright-field TEM image of the complete lamella, f) bright-field

TEM image of dense bond between polished $\mathrm{Al}$ substrate and PA, g) bright-field image of individual $\mathrm{Al}_{\mathrm{x}} \mathrm{O}_{\mathrm{y}}$ nanostructures embedded in $\mathrm{PA}$, and $\mathrm{h}$ ) highresolution bright-field image of $\mathrm{Al}_{\mathrm{x}} \mathrm{O}_{\mathrm{y}}$ nanostructures in amorphous PA.

\section{Conclusions}

Based on the presented study on the effect of different aluminum pretreatments on the mechanical properties of the laser-welded aluminum/polyamide specimens, the followings can be concluded:

- As-received Al samples show anisotropy of surface roughness. Changing the Al samples orientation (welding to PA in rolling or perpendicular to the rolling direction) does not have a major effect on the shear loads of the joint.

- By increasing the Ra value with the abrasive blasting process, the shear loads of the joint significantly reduce due to high thermal contact resistance. For high $\mathrm{Ra}$ samples, there is practically no durable joint between $\mathrm{Al}$ and PA, and the PA residue on the $\mathrm{Al}$ surface after failure is negligible.

- The negative effect of high thermal contact resistance is observed for laser-ablated high $\mathrm{Ra}$ samples, while for medium Ra samples there is a significant improvement in the shear load of the joints compared to as-received condition.
- The highest shear load (improvement of approximately $58 \%$ compared to the as-received condition) is observed for laser-polished under atmospheric environment samples thanks to the presence of the artificial nano-structured $\mathrm{Al}$ oxide layer. The PA residue on the Al surface after failure is relatively thick and uniform which indicated a good adhesion between $\mathrm{Al}$ and PA surfaces.

- By comparing the observation and results for the different pretreatment processes, by promoting the physicochemical bonding, a superior joint is achieved.

\section{References}

[1] Moldovan, E., Tierean, M.H., Stanciu, E.M. (2017) Overview of Joining Dissimilar Materials: Metals and Polymers, Bulletin of the Transilvania University of Braşov 10, 39-46.

[2] Farazila, Y., Fadzil, M., Hamdi, M. (2012) A Brief Review: Laser Joining of Polymer-Metal Structures, ASEAN Engineering Journal 2, 5-12.

[3] Katayama, S., Kawahito, Y. (2008) Laser direct joining of metal and plastic, Scripta Materialia 59, 1247-1250.

[4] Kawahito, Y., Niwa, Y., Katayama, S. (2014) Laser direct joining between stainless steel and polyethylene terephthalate plastic and reliability evaluation of joints, Welding International 28,107-113.

[5] Heckert, A., Zaeh, M.F. (2014) Laser surface pretreatment of aluminium for hybrid joints with glass fibre reinforced thermoplastics. Physics Procedia 56, 11711181.

[6] Heckert, A., Singer, C., Zaeh, M.F. (2015) Pulsed Laser Surface Pre-Treatment of Aluminium to Join Aluminium- Thermoplastic Hybrid Parts, Lasers in Manufacturing Conference (LiM).

[7] Schricker, K., Stambke, M., Bergmann, J.P., Bräutigam, K., Henckell, P. (2014) Macroscopic surface structures for polymer-metal hybrid joints manufactured by laser based thermal joining. Physics Procedia 56, 782-790.

[8] Amne Elahi, M., Koch, M., Heck, M., Plapper, P. (2020) Pre and post-treatments to improve weldability and mechanical properties of Aluminum-Polyamide laser welded specimens, Procedia CIRP 94, 537-541. 
[9] Grujicic, M., Sellappan, V., Omar, M.A., Seyr, N., Obieglo, A., Erdmann, M., Holzleitner, J. (2008) An overview of the polymer-to-metal direct-adhesion hybrid technologies for load-bearing automotive components, Journal of Materials Procecessing Technology 197, 363-373.

[10] Yovanovich, M.M. (2005) Four decades of research on thermal contact, gap, and joint resistance in microelectronics, IEEE Transactions on Components and Packaging Technologies 28, 182-206.

[11] Amne Elahi, M., Koch, M., Plapper, P. (2019) Laser polishing of Aluminum and Polyamide for dissimilar laser welded assemblies, Lasers in Manufacturing Conference (LiM).

\section{Acknowledgment}

The presented work is based on "Process Innovation for Sensors in Mobile Applications Based on Laser Assisted Metal-Plastic Joining" project (AFR-PPP grant, Reference 11633333). The authors would like to thank Birgit Nothdurft (INM) for excellent FIB sample preparation, the support of the Luxembourg National Research Fund (FNR), and acknowledge Cebi Luxembourg S.A. as the industrial partner of the project.

\section{Meet the Author}

Mahdi Amne Elahi holds M.Sc. degree in Materials Engineering from Iran University of Science and Technology with few years of working background as the head of quality control and laboratories in aluminum flat-rolled production. He is currently a Ph.D. student at the University of Luxembourg. His research is focused on laser surface treatment and the welding of dissimilar materials.

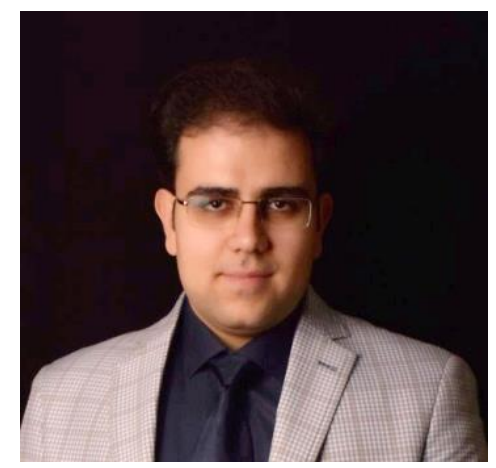

